\title{
Convergence of patient- and physician-reported outcomes in the French National Registry of Facioscapulohumeral Dystrophy
}

Benoît Sanson ${ }^{*} \mathbb{0}$, Caroline Stalens ${ }^{2}$, Céline Guien ${ }^{3}$, Luisa Villa ${ }^{1}$, Catherine Eng ${ }^{2}$, Sitraka Rabarimeriarijaona ${ }^{4}$, Rafaëlle Bernard ${ }^{4}$, Pascal Cintas ${ }^{5}$, Guilhem Solé6 ${ }^{6}$ Vincent Tiffreau ${ }^{7}$, Andoni Echaniz-Laguna ${ }^{8,9,10}$, Armelle Magot ${ }^{11}$, Raul Juntas Morales ${ }^{12}$, François Constant Boyer ${ }^{13}$, Aleksandra Nadaj-Pakleza ${ }^{14,15}$, Agnès Jacquin-Piques ${ }^{16}$, Christophe Béroud ${ }^{3,4+}$, Sabrina Sacconi ${ }^{1,17 \dagger}$ and The French FSHD registry collaboration group

\begin{abstract}
Background: Facioscapulohumeral muscular dystrophy (FSHD) is among the most prevalent muscular dystrophies and currently has no treatment. Clinical and genetic heterogeneity are the main challenges to a full comprehension of the physiopathological mechanism. Improving our knowledge of FSHD is crucial to the development of future therapeutic trials and standards of care. National FSHD registries have been set up to this end. The French National Registry of FSHD combines a clinical evaluation form (CEF) and a self-report questionnaire (SRQ), filled out by a physician with expertise in neuromuscular dystrophies and by the patient, respectively. Aside from favoring recruitment, our strategy was devised to improve data quality. Indeed, the pairwise comparison of data from 281 patients for 39 items allowed for evaluating data accuracy. Kappa or intra-class coefficient (ICC) values were calculated to determine the correlation between answers provided in both the CEF and SRQ.

Results: Patients and physicians agreed on a majority of questions common to the SRQ and CEF (24 out of 39). Demographic, diagnosis- and care-related questions were generally answered consistently by the patient and the medical practitioner (kappa or ICC values of most items in these groups were greater than 0.8). Muscle functionrelated items, i.e. FSHD-specific signs, showed an overall medium to poor correlation between data provided in the two forms; the distribution of agreements in this section was markedly spread out and ranged from poor to good. In particular, there was very little agreement regarding the assessment of facial motricity and the presence of a winged scapula. However, patients and physicians agreed very well on the Vignos and Brooke scores. The report of symptoms not specific to FSHD showed general poor consistency.
\end{abstract}

Conclusions: Patient and physician answers are largely concordant when addressing quantitative and objective items. Consequently, we updated collection forms by relying more on patient-reported data where appropriate. We

\footnotetext{
*Correspondence: sanson.b@chu-nice.fr

${ }^{\dagger}$ Christophe Béroud and Sabrina Sacconi have contributed equally

1 Université Côte d'Azur, Service Système Nerveux Périphérique \& Muscle,

Centre Hospitalier Universitaire de Nice, Nice, France

Full list of author information is available at the end of the article
}

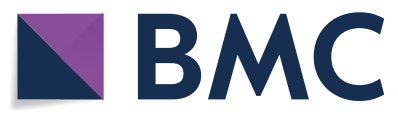

(c) The Author(s) 2022. Open Access This article is licensed under a Creative Commons Attribution 4.0 International License, which permits use, sharing, adaptation, distribution and reproduction in any medium or format, as long as you give appropriate credit to the original author(s) and the source, provide a link to the Creative Commons licence, and indicate if changes were made. The images or other third party material in this article are included in the article's Creative Commons licence, unless indicated otherwise in a credit line to the material. If material is not included in the article's Creative Commons licence and your intended use is not permitted by statutory regulation or exceeds the permitted use, you will need to obtain permission directly from the copyright holder. To view a copy of this licence, visit http://creativecommons.org/licenses/by/4.0/. The Creative Commons Public Domain Dedication waiver (http://creativeco mmons.org/publicdomain/zero/1.0/) applies to the data made available in this article, unless otherwise stated in a credit line to the data. 
hope the revised forms will reduce data collection time while ensuring the same quality standard. With the advent of artificial intelligence and automated decision-making, high-quality and reliable data are critical to develop topperforming algorithms to improve diagnosis, care, and evaluate the efficiency of upcoming treatments.

\section{Background}

Facioscapulohumeral Dystrophy (FSHD) is one of the most common dystrophies in adults. The prevalence of the disease has been reported ranging from $\sim 1: 8000$ to $\sim 1: 15,000$ in the US $[1,2], \sim 1: 8000$ in the Netherlands [3], and 1:20,000 in Italy [4] and the UK $[5,6]$. No epidemiological study has been performed in France yet, but by conservative standards, it can be estimated that at least 3500 people carry the disease.

FSHD is characterized by progressive asymmetric muscle weakness, with early involvement of facial muscles, progressive weakness and atrophy of scapular and humeral muscles, and later involvement of the trunk and lower extremities. The disease shows significant interand intra-familial clinical variability in terms of progression and severity. Disease onset is usually before the second decade; early onset is associated with faster progression and higher severity as most wheelchair-bound FSHD patients have had childhood onset of the disease $[7,8]$. These severe FSHD patients are more prone to develop an extra muscular complication of the disease, such as central nervous system involvement $[9,10]$, retinal telangiectasia [11], and hearing impairment [12].

FSHD is associated with epigenetic derepression of D4Z4 repeats on chromosome 4q. The common form FSHD type 1 (FSHD1; 95\% of patients) is associated with a pathogenic contraction of D4Z4 repeat units (RUs; 1-10). Patients with the rare form FSHD type 2 (FSHD2) have more than 10 D4Z4 RUs combined with defects in D4Z4 chromatin repressors, mostly SMCHD1 gene mutations [13]. It has recently been suggested that the two types of the disease correspond to a genetic and epigenetic continuum [14]. In both FSHD types, the aberrant expression of the D4Z4-encoded DUX4 gene has been proposed to cause the disease through a toxic gainof-function mechanism [15].

Genetic and clinical heterogeneity [16] in FSHD may complicate diagnosis and proper genetic counseling, and prevent the development of clinical, biological and patient-reported outcome measures (PROMs) to evaluate disease severity and progression, and the efficacy of therapeutic strategies.

Until recently, data on FSHD were scarce. To ease data collection on FSHD, national registries have been, or are being, set up in Europe, Northern America, Egypt, Australia, and New Zealand [6, 8, 17-19]. While efforts have been made to ensure a common data set [20], the objectives of such databases are multiple. For instance, the Italian registry [18] predominantly aims at gaining novel insights into the natural history of the disease. In contrast, the US [8], UK [6], and New Zealand [17] registries have been designed as tools to ease the enrollment of patients in clinical trials and studies. The French FSHD registry has been collecting data since 2013, and purposed both with a better understanding of the natural course of FSHD and the facilitation of clinical trials, e.g. by identifying PROMs or tailoring eligibility criteria.

Data are often gathered through clinical evaluation, which relies on the active participation of medical practitioners. Alternatively, some databases aggregate patientreported data. However, the ability of FSHD patients in collecting data on their disease has never been investigated. Most registries use a single questionnaire, filled out either by the physician or by the patient. Except for the US registries, where a minority of questions are addressed to the clinician, all registries collect data from a single source. As it is unlikely that a single rater best assesses all relevant registry items, the French FSHD registry was designed to gather information from both the patient with a self-report questionnaire (SRQ) and the neurologist with a clinical evaluation form (CEF) [19]. The objective was to not only reach a greater population, but also to assess and improve data accuracy and quality, which is critical in order to harmonize and share data at the international level, thus enabling machine-learning (ML) and AI approaches.

This study evaluates the concordance between SRQs and CEFs used in the French FSHD registry in a cohort of 281 patients to optimize these forms for high-quality data collection.

\section{Patients and methods}

\section{Patient cohort}

Data were collected using predesigned forms: an SRQ and a CEF (available on the registry website, www.fshd. fr) [19] completed by the patient and the neurologist, respectively. FSHD1 patients of the French National Registry of FSHD were included in the analysis, provided both forms were available. Additionally, the two forms were to be completed within a period of 3 months, which was deemed short enough to prevent a significant evolution of clinical signs and symptoms between the two assessments. Among the 605 patients included in the registry at the time of the analysis (September 2017), 281 
patients were selected. Fig 1 shows the flowchart of the selection process. Signed informed consent was obtained from each patient before any data collection. The relevant national ethics committees approved the registry, namely the French data protection authority (CNIL; Authorization Number 912291) and the French advisory committee on data processing in health research (CCTIRS; Favorable Opinion Number 12.004bis).

\section{Data collected}

Items collected in both the SRQ and the CEF were analyzed to evaluate internal consistency between the two raters (patient and clinician). The forms enclose 42 mutual items, out of which 39 could be statistically assessed in a relevant manner (Fig. 2 and Table 1). The 39 compared items are divided into seven sections: diagnosis, demographics, muscle function, care; as well as heart, respiratory and GI symptoms. It should be noted that the SRQ asked for a self-evaluation of muscle function. Indeed, patients were proposed to self-grade their arm and leg function using reformulated Brooke [21] and Vignos [22] scales; physicians usually perform such scoring. Patients were also asked to self-evaluate facial involvement through yes/no questions such as "Are you able to whistle?" or "Do you have difficulty closing your eyes?".

\section{Statistical analysis}

For qualitative items, an inter-rater reliability analysis using Cohen's kappa statistic was performed. The definition is $K=\frac{p_{o}-p_{e}}{1-p_{e}}$, where $\mathrm{p}_{\mathrm{o}}$ is the relative observed agreement among raters and $\mathrm{p}_{e}$ is the relative agreement among raters expected by chance. The interpretation was performed using the Landis and Koch guidelines [23], while considering the known limitations of the kappa statistic, such as the prevalence of observations and heterogeneous margins $[24,25]$.

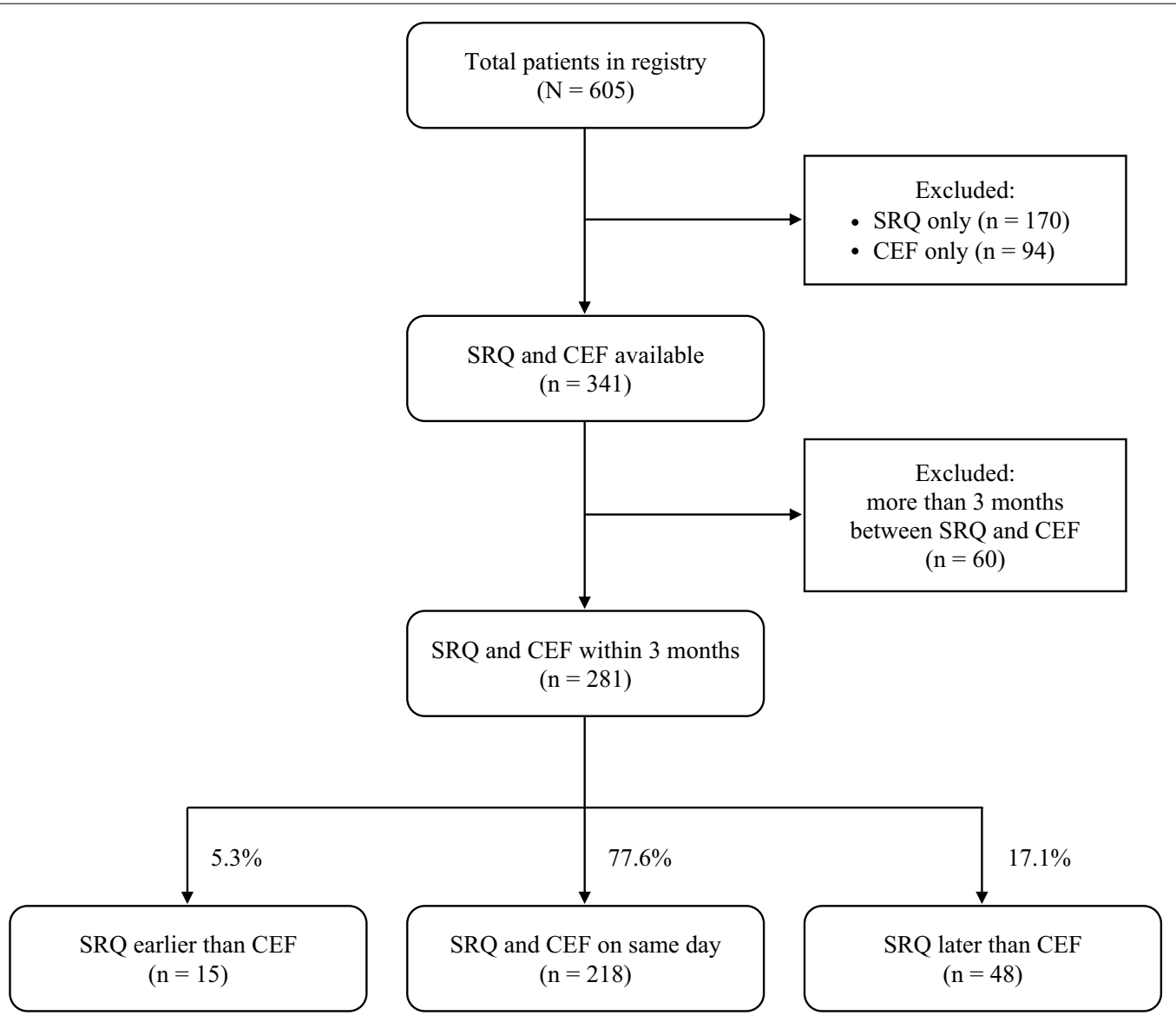

Fig. 1 Patient selection process in the analysis. The distribution of selected patients, i.e., having both a self-report questionnaire (SRQ) and a clinical evaluation form (CEF) filled within 3 months at the time of analysis, is shown according to the order of completion of forms 


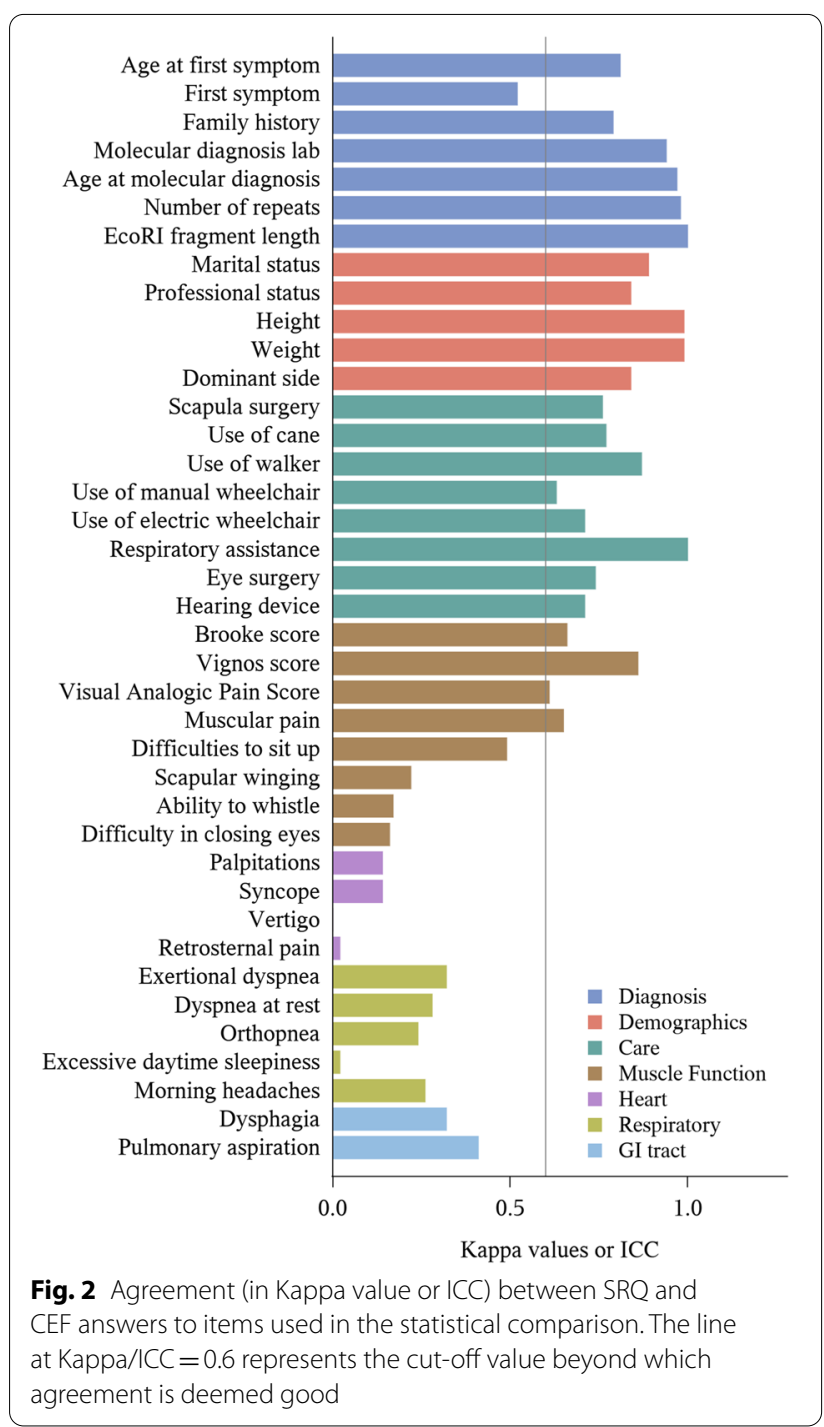

For quantitative items, intraclass correlation coefficients (ICC) were used. We considered a "two-way random-effects" model as we planned to generalize our reliability results to any rater possessing the same characteristics as those selected (patient or clinician) and a "single rater/measurement" type as we planned to use the measurement from a single rater as the basis of the actual measurement [26]. The degree of consistency is $\hat{\rho}=\frac{M S_{R}-M S_{E}}{M S_{R}+M S_{E}}$, where $\mathrm{MS}_{\mathrm{R}}$ and $M S_{\mathrm{E}}$ are the mean squares for rows and error, respectively. The ICC interpretation was performed according to the criteria used for that of the kappa coefficient.

The statistical analysis was performed using the SAS software, version 9.4 (SAS Institute Inc., Cary, NC, USA).

\section{Subgroup analyses}

The same analysis was performed separately on data split into two groups according to a series of parameters: gender, age ( $<65$ years vs. $\geq 65$ years), education level (pre- vs. post- French secondary education degree), and disease severity (Clinical Severity Score $[27]<6$ vs. $\geq 6$ ).

\section{Results}

\section{Description of the cohort}

The cohort consisted of 131 women and 150 men with a mean age of $54.8 \pm 16.0$ years (Table 2). With a number of RUs ranging from 2 to 10 and $11 \%$ of non-ambulatory patients, the cohort can be deemed representative of the general FSHD population.

As shown in Fig. 1, the SRQ and CEF were filled out on the same day in most cases (77.6\%). SRQs were filled out later than CEFs more often than earlier $(17.1 \%$ vs. $5.3 \%$, respectively). The mean time between the filling out of two forms was 0.62 days \pm 11.82 days (data not shown).

From this point on, all the following results pertain to items with an answer in both the SRQ and the CEF. Among the response pairs, the age at onset, completed in both forms for $84 \%$ of patients ( 236 out of 281 ), varied from 0 to 75 years in the SRQ, and from 1 to 73 years in the CEF (Table 3). The first symptoms appeared on average in the late twenties, with a mean age of $28.6 \pm 17.4$ years in the SRQ and $26.9 \pm 16.6$ years in the CEF. They were primarily localized in the proximal end of the superior limb: $51 \%$ and $55 \%$, according to patients and neurologists, respectively, out of 255 answer pairs (Table 3).

\section{Comparative analysis of patient and physician assessments} For each of the 39 analyzable items, the consistency between the responses in the two types of forms was evaluated (Table 1 and Fig. 2). Specific results per item category are given below.

\section{Diagnosis}

Most items related to diagnosis, i.e. related to first symptoms or genetics, showed excellent consistency between patient and physician reporting (kappa value higher than 0.8 ). Interestingly, the age at first symptoms was highly consistent (ICC equal to 0.81 ). Most genetic-related items, namely the laboratory where the analysis was performed, the age at the time of molecular diagnosis, the number of RUs, and the EcoRI fragment length, were nearly identical in form pairs (kappa value or ICC of 0.94, 0.97, 0.98 and 1, respectively). Moreover, the consistency of the item "family history" was also relatively high (kappa of 0.79). However, the description of the first symptom is an outlier 
Table 1 Inter-rater agreement, evaluated as a percentage agreement, and as a kappa value or ICC, for the 39 items common to the SRQ and CEF used in the statistical comparison; class sizes are provided and items are grouped by sections

\begin{tabular}{|c|c|c|c|c|c|}
\hline Section & Item & $\mathrm{n}$ & PA (\%) & Kappa $[95 \%$ Cl] & ICC $[95 \%$ Cl] \\
\hline \multirow[t]{7}{*}{ Diagnosis } & Age at first symptom & 236 & - & - & $0.81[0.76-0.85]$ \\
\hline & First symptom & 255 & 0.68 & $0.52[0.44-0.60]$ & - \\
\hline & Family history & 266 & 0.90 & $0.79[0.72-0.87]$ & - \\
\hline & Molecular diagnosis lab & 253 & 0.97 & $0.94[0.90-0.98]$ & - \\
\hline & Age at molecular diagnosis & 236 & - & - & $0.97[0.96-0.98]$ \\
\hline & Number of repeats & 219 & - & - & $0.98[0.97-0.98]$ \\
\hline & EcoRl fragment length & 8 & - & - & $1.00[-]^{*}$ \\
\hline \multirow[t]{5}{*}{ Demographics } & Marital status & 270 & 0.93 & $0.89[0.84-0.93]$ & - \\
\hline & Professional status & 272 & 0.89 & 0.84 [0.79-0.89] & - \\
\hline & Height & 271 & - & - & 0.99 [0.99-0.99] \\
\hline & Weight & 266 & - & - & 0.99 [0.99-0.99] \\
\hline & Dominant side & 268 & 0.96 & $0.84[0.75-0.93]$ & - \\
\hline \multirow[t]{8}{*}{ Care } & Scapula surgery & 276 & 0.97 & $0.76[0.61-0.92]$ & - \\
\hline & Use of cane & 281 & 0.93 & $0.77[0.67-0.86]$ & - \\
\hline & Use of walker & 281 & 0.98 & $0.87[0.77-0.96]$ & - \\
\hline & Use of manual wheelchair & 281 & 0.92 & $0.63[0.49-0.77]$ & - \\
\hline & Use of electric wheelchair & 281 & 0.93 & $0.71[0.58-0.83]$ & - \\
\hline & Respiratory assistance & 19 & 1.00 & $1.00[1.00-1.00]$ & - \\
\hline & Eye surgery & 40 & 0.88 & $0.74[0.54-0.95]$ & - \\
\hline & Hearing device & 30 & 0.87 & $0.71[0.46-0.97]$ & - \\
\hline \multirow[t]{8}{*}{ Muscle function } & Brooke scale & 254 & - & - & $0.66[0.58-0.72]$ \\
\hline & Vignos scale & 250 & - & - & $0.86[0.82-0.89]$ \\
\hline & Visual Analog Pain Score (VAPS) & 191 & - & - & $0.61[0.51-0.69]$ \\
\hline & Muscular pain & 270 & 0.89 & $0.65[0.53-0.76]$ & - \\
\hline & Difficulties to sit up & 263 & 0.80 & $0.49[0.37-0.61]$ & - \\
\hline & Scapular winging & 241 & 0.86 & $0.22[0.06-0.38]$ & - \\
\hline & Ability to whistle & 271 & 0.55 & $0.17[0.10-0.25]$ & - \\
\hline & Difficulties in closing eyes & 272 & 0.46 & $0.16[0.10-0.21]$ & - \\
\hline \multirow[t]{4}{*}{ Heart } & Palpitations & 281 & 0.74 & $0.14[0.04-0.23]$ & - \\
\hline & Syncope & 281 & 0.92 & $0.14[-0.04-0.32]$ & - \\
\hline & Vertigo & 281 & 0.77 & $0.00[0.00-0.00]$ & - \\
\hline & Retrosternal pain & 281 & 0.81 & $0.02[-0.05-0.08]$ & - \\
\hline \multirow[t]{5}{*}{ Respiratory } & Exertional dyspnea & 281 & 0.71 & $0.32[0.21-0.43]$ & - \\
\hline & Dyspnea at rest & 281 & 0.92 & $0.28[0.06-0.49]$ & - \\
\hline & Orthopnea & 281 & 0.94 & $0.24[0.01-0.47]$ & - \\
\hline & Excessive daytime sleepiness & 281 & 0.79 & $0.02[-0.07-0.12]$ & - \\
\hline & Morning headaches & 281 & 0.88 & $0.26[0.10-0.42]$ & - \\
\hline \multirow[t]{2}{*}{ Gl tract } & Dysphagia & 281 & 0.85 & $0.32[0.16-0.47]$ & - \\
\hline & Pulmonary aspiration & 281 & 0.85 & $0.41[0.27-0.55]$ & - \\
\hline
\end{tabular}

$n$ number of observations, PA percentage of agreement, ICC intraclass correlation coefficient, $\underline{C l}$ confidence interval, VAPS visual analog pain scale

* $\mathrm{Cl}$ not computed because the number of observations was too small

in this section as it was associated with a medium consistency (kappa of 0.52). The prevalence of all items in this section was between 219 and 266, except for the EcoRI fragment length, which was completed in only eight form pairs. It is also worth noting that the number of RUs was answered significantly more often by the physician (255; data not shown) than the patient (219).

\section{Demographics}

As expected, all items in the demographic section showed a good agreement between the patient and the 
Table 2 Statistics description of the cohort

\begin{tabular}{ll}
\hline Characteristic & $\mathbf{n}=\mathbf{2 8 1}$ \\
\hline Gender & \\
$\quad$ Male & $150(53.4 \%)$ \\
$\quad$ Female & $131(46.6 \%)$ \\
Age (years) & \\
$\quad$ Mean \pm SD & $54.8 \pm 16.0$ \\
$\quad$ Range & $21-96$ \\
D4Z4 repeat array size (units) & $2-10$ \\
Ability to walk & \\
Yes & $250(89.0 \%)$ \\
No & $31(11.0 \%)$ \\
\hline
\end{tabular}

$S D$ standard deviation

Table 3 Clinical diagnosis data recorded by form type

\begin{tabular}{lll}
\hline Characteristic & $\begin{array}{l}\text { Self-report } \\
\text { questionnaire }\end{array}$ & $\begin{array}{l}\text { Clinical } \\
\text { evaluation } \\
\text { form }\end{array}$ \\
\hline $\begin{array}{l}\text { Age at onset }(\mathrm{n}=236) \\
\quad \text { Mean } \pm \text { SD }\end{array}$ & $28.6 \pm 17.4$ & $26.9 \pm 16.6$ \\
$\quad$ Range (years) & $0-75$ & $1-73$ \\
First symptom ( $\mathrm{n}=255)$ & & $2(0.8 \%)$ \\
Asymptomatic patient & $2(0.8 \%)$ & $38(15 \%)$ \\
Facial involvement & $41(16 \%)$ & $139(55 \%)$ \\
Proximal upper limb involvement & $130(51 \%)$ & $7(2.7 \%)$ \\
Distal upper limb involvement & $2(0.8 \%)$ & $27(11 \%)$ \\
Proximal lower limb involvement & $41(16 \%)$ & $25(9.8 \%)$ \\
Distal lower limb involvement & $10(3.9 \%)$ & $17(6.7 \%)$ \\
Other symptom & $28(11 \%)$ & \\
\hline
\end{tabular}

physician (kappa or ICC greater than or equal to 0.84; Table 1). Height and weight, in particular, had a very high agreement (ICC of 0.99). Though slightly lower, dominant side, and marital and employment statuses also showed excellent consistency (kappa values of 0.84, 0.89, and 0.84 , respectively). With a prevalence greater than or equal to 266 (out of a cohort of 281), demographic items were reported in nearly all form pairs.

\section{Medical care}

The data indicated a good to excellent agreement for all items related to surgical procedures and the use of a medical device. Indeed, the items related to scapula and eye surgery, and the use of a hearing aid and most walking devices listed in the forms (cane, manual and electric wheelchairs), showed a good agreement (kappa values between 0.63 and 0.77). Furthermore, the items "use of a walker" and "respiratory assistance" showed excellent agreement (kappa values of 0.87 and 1 , respectively). The prevalence of all items in this section was very high, except for "respiratory assistance", "hearing aid" and "eye surgery" $(19,30$, and 40 , respectively). The only caveat is that a prevalence of 281 was systematically observed for the 15 items related to multiple-choice questions where "no" was not a proposed choice. Indeed, owing to the data structure, the absence of an answer was then indistinguishable from "none" or "no".

\section{Muscle function}

The prevalence of all items in this section was greater than or equal to 241, except for that related to the visual analog pain score (VAPS) with 191 (see Table 1). The distribution of agreements was very diffuse. On the one hand, the agreement between patients and physicians regarding body motricity was good to very good, except for the item "difficulties to sit up", which showed a medium agreement. Indeed, the item related to the Vignos score, which evaluates the lower extremity function on a scale from 1 to 10,1 being the least severe involvement, showed a very high agreement (ICC of 0.86). Additionally, the Brooke score, which is the upper extremity counterpart of the Vignos score (on a scale from 1 to 6), as well as the items "muscular pain" and "Visual Analog Pain Score (VAPS)", yielded good agreements (kappa value or ICC between 0.61 and 0.66). On the other hand, patients and physicians mostly disagreed when assessing facial motricity ("difficulty in closing eyes" and "ability to whistle") and "scapular winging" (kappa values between 0.16 and 0.22 ).

Interestingly, the item "difficulty in closing eyes" was predominantly answered negatively by patients $(76 \%$ of SRQs; Table 4) but positively by physicians (72\% of CEFs). It is striking that most of the related discordance (50\% of answer pairs) was associated with patients answering "no" in the SRQ but having a counterpart "yes" in the matching CEF. The opposite situation ("yes" in the SRQ and "no" in the CEF") was observed in only $1.1 \%$ of cases. The item "ability to whistle" showed a similar discrepancy, although in reverse (a majority of "yes" in the SRQ matched by a "no" in the CEF) and with more balanced associated SRQ answers. The item "scapular winging" exhibited a similar trend but to a more limited extent. Although the agreement was low, the percentage of agreement was still relatively high $(86 \%$; Table 1$)$.

One of the aforementioned items is formulated as an ability while the two others as a difficulty. The disagreements observed in the facial motricity-related and "scapular winging" items were thus similar: in most cases, when answers did not match, the observed disagreement was one-way. More specifically, the absence of a report of 
Table 4 Breakdown of response pairs, in the CEF and SRQ, to four discordant items

\begin{tabular}{|c|c|c|c|c|c|}
\hline Item & SRQ answer & CEF answer & & & \\
\hline \multirow[t]{5}{*}{ Difficulty in closing eyes } & & Uncertain & No & Yes & Total \\
\hline & Uncertain & $\overline{0(0.0 \%)}$ & $0(0.0 \%)$ & $1(0.4 \%)$ & $1(0.4 \%)$ \\
\hline & No & $8(2.9 \%)$ & $65(24 \%)$ & $135(50 \%)$ & $208(76 \%)$ \\
\hline & Yes & $0(0.0 \%)$ & $3(1.1 \%)$ & $60(22 \%)$ & $63(23 \%)$ \\
\hline & Total & $8(2.9 \%)$ & $68(25 \%)$ & $196(72 \%)$ & $272(100 \%)$ \\
\hline \multirow[t]{5}{*}{ Ability to whistle } & & Uncertain & No & Yes & Total \\
\hline & Uncertain & $0(0.0 \%)$ & $2(0.7 \%)$ & $0(0.0 \%)$ & $2(0.7 \%)$ \\
\hline & No & $1(0.4 \%)$ & $114(42 \%)$ & $7(2.6 \%)$ & $122(45 \%)$ \\
\hline & Yes & $2(0.7 \%)$ & $109(40 \%)$ & $36(13 \%)$ & $147(54 \%)$ \\
\hline & Total & $3(1.1 \%)$ & $225(83 \%)$ & $43(16 \%)$ & $271(100 \%)$ \\
\hline \multirow[t]{4}{*}{ Winged scapula } & & & No & Yes & Total \\
\hline & No & & $6(2.5 \%)$ & $30(12 \%)$ & $36(15 \%)$ \\
\hline & Yes & & $3(1.2 \%)$ & $202(84 \%)$ & $205(85 \%)$ \\
\hline & Total & & $9(3.7 \%)$ & $232(96 \%)$ & $241(100 \%)$ \\
\hline \multirow[t]{4}{*}{ Vertigo } & & & No & Yes & Total \\
\hline & No & & $216(77 \%)$ & $0(0.0 \%)$ & $216(77 \%)$ \\
\hline & Yes & & $65(23 \%)$ & $0(0.0 \%)$ & $65(23 \%)$ \\
\hline & Total & & $281(100 \%)$ & $0(0.0 \%)$ & 281 (100\%) \\
\hline
\end{tabular}

$S R Q$ self-report questionnaire, CEF clinical evaluation form

FSHD-specific symptoms in the CEF was rarely matched with a report in the SRQ. On the contrary, the report of such symptoms in the CEF was comparably associated with either a report or an absence thereof in the SRQ.

\section{Signs and symptoms not specific to FSHD}

The agreement of items related to signs and symptoms not specific to FSHD, included in the analysis, ranged from very poor to medium. A poor to very poor agreement (kappa value between 0 and 0.32) was observed for all items related to heart and respiratory signs and symptoms. GI tract-related items "dysphagia" and "pulmonary aspiration" showed a low to medium agreement (kappa value of 0.32 and 0.41 respectively). In contrast, the prevalence was very high and equal to 281 for all items, with the caveat mentioned above.

The item "vertigo and dizziness" showed a peculiar phenomenon, with patients much more prone to report symptoms than physicians. Strikingly, no positive answer was recorded in the CEFs, although $23 \%$ of SRQs recorded "yes" (Table 4). A similar observation was made with "retrosternal pain", where the CEFs of nearly all patients (99\%) reported a "no" but $18 \%$ positive answers were found in the SRQs (data not shown). Hence, positive responses in the CEF were scarcely matched with a negative answer in the SRQ, i.e. the mismatch happened one way, not the other, suggesting that specific symptoms were not necessarily wrongly identified but may have been overlooked by physicians or overestimated by patients. The two items mentioned above displayed the lowest agreement in the analysis (kappa values of 0 and $0.02 \%$ for vertigo and retrosternal pain, respectively).

Overall, symptoms reported in the SRQ were not systematically reported by the neurologist. The symptoms most frequently unparalleled by the physician in the CEF were vertigo, retrosternal pain, and daytime sleepiness. The unparalleled reporting of symptoms between form types was further analyzed by center, yielding no significant difference (data not shown).

\section{Summary}

Items corresponded to either quantitative or qualitative variables. All quantitative variables yielded high agreement (ICC greater than 0.6). Qualitative variables did not display a specific trend in agreement (kappa values scattering the whole range from 0 to 1 ).

\section{Effect of age, sex, education level, and disease severity on rates of agreement}

The cohort was split into two groups according to a predefined cut-off in each category to assess the effects of age, sex, education level, and disease severity. The statistical comparison performed on these groups did not significantly differ relative to the general cohort (Additional file 1: Figure S1, Additional file 2: Figure S2, Additional file 3: Figure S3, Additional file 4: Figure S4, Additional 
file 5: Figure S5, Additional file 6: Figure S6, Additional file 7: Figure S7, Additional file 8: Figure S8).

\section{Discussion}

\section{Cohort representativeness}

In this study, we compared data from SRQs and CEFs. Data were analyzed only if both forms were available and had been filled out within a 3-month period, to mitigate variations due to disease evolution. Overall, data from the whole registry and our selected cohort of 281 patients were consistent with what was observed in epidemiological studies [1-6]. Indeed, $95.01 \%$ of patients in the French registry had FSHD1 at the time of analysis, perfectly reflecting the finding that type 1 represents at least $95 \%$ of cases [28]. Besides, in the selected cohort, the first symptoms appeared on average in the late twenties, as previously described [29]. The consistency of the cohort with preexisting epidemiological data can be further established. For instance, the initial symptom most often reported in the French registry, and the cohort, was an involvement of the proximal upper limb muscles, as described in several epidemiological studies [6]. Another evidence can be found in the broad distribution of ages (21-99), and nearly complete FSHD1-compatible range of RUs (2-10) observed in the study cohort.

\section{Concordance of patient- and physician-reported data}

The comparison of the answers provided by patients and physicians on 39 items allowed assessing the inter-rater reliability of the data reported. To our knowledge, it is the first time that the agreement of patient- and physicianreported data has been evaluated in a neuromuscular disease registry. The present study was made possible by the dual data collection strategy set forth in the French FSHD registry.

Our results showed that patients and physicians agreed in most cases. Indeed, a majority of items analyzed (24 out of 39) showed good to excellent agreement. Most questions found in the demographic, diagnosis, muscle function, and care sections were answered identically by the physician and the patient. It follows that the corresponding PROMs are as trustworthy as the assessments made by the physician, and the related questions thus need not necessarily be asked to the latter to collect a full dataset on a patient at a given time. As the medical consultation time is limited, it is highly desirable to optimize the CEF by either shortening it as much as possible or replacing superfluous items with assessments requiring medical expertise. The paramount importance of patients in contributing to the registry through self-reporting data is herein evidenced.

\section{Patients and physicians disagree on symptoms}

Patients and physicians gave discordant answers regarding most signs and symptoms. The structure of the discordance suggests that patients tend to ignore or minimize the impairments the FSHD-specific symptoms are related to, which is in accord with our experience and has been described in patients with oculopharyngeal muscular dystrophy [30]. However, symptoms related to comorbidities showed a different behavior. In contrast to FSHD-specific signs, patients tended to report symptoms not specific to FSHD more readily than physicians. Some symptoms may require a trained specialist to be recognized but are more likely to be overlooked by said specialist when filling out a time-consuming comprehensive form. Therefore, no single data source should be privileged in this case. Even though the physician answers may be more accurate, the patient information is more complete. Comorbidity-related symptoms should thus be collected in both forms.

\section{Limitations and biases}

Although concordant when answered in both forms, several items, such as genetic information, were moderately to scarcely reported by patients, likely owing to their inherent technicality. Such items are therefore best left to the physician. It is worth noting that the number of RUs and fragment length are two sides of the same coin; either information was sufficient, thereby deterring physicians from providing redundant information. The fragment length may thus be removed from future forms.

The order in which SRQs and CEFs were completed likely influenced the answers provided in either form. The influence of each rater on the other thus cannot be ruled out. Furthermore, potential help from the staff in completing either form probably drove up concordance. However, although representing a statistical bias in the present study, such an effect actually underscores the effectiveness of using both patient- and physicianreported forms by helping collect complete and reliable datasets.

Data reported in the CEFs were carefully monitored, except when related to symptoms. Moreover, physicians are trained to assess outcome measures. In this light, we could reasonably, albeit roughly, assume the reliability of objective physician-reported data. In the following, for practical reasons, we thus equated inter-rater concordance to patient reliability regarding relevant items.

\section{Optimization of data collection forms}

The concordance of responses is highly dependent on the nature of the question and the collection modality. Our analysis showed that data can reliably be collected 
directly by the patient, provided it is straightforward, objective, or quantitative. In particular, the surprisingly good agreements of the Vignos and Brooke scales showed that patients could accurately answer detailed, technical questions. In contrast to the description of symptoms, the lower and upper limb functional assessment scales are defined in simple words. The general higher consistency of quantitative variables, therefore, drives us to employ quantifiable items whenever possible. The formulation of questions is also essential. Notably, medical jargon should be avoided in SRQs.

The availability of physicians is a limiting factor when collecting data for a registry. This is also true, to a lesser extent, for patients who are generally asked to fill many questionnaires when visiting their FSHD doctor. The registry questionnaires must therefore be as short as possible. However, as the natural history and causes of FSHD are still largely being explored, relevant questions are many. To minimize the burden for both raters, we propose not to ask clinical or technical questions to patients unless it has an objective or quantifiable aspect, such as the Vignos and Brooke scales.

Conversely, all questions that do not specifically require medical training can be left to the patient. Nevertheless, a number of such questions should be asked in both forms as an internal control to further assess the reliability of answers provided and pursue the optimization of the forms. Furthermore, it can help collect data that physicians will not provide for lack of time, e.g. data related to non-FSHD symptoms, even though the reliability of which cannot be assumed (with the limitations mentioned above) as positively as that of objective data. Besides, both forms are not available for all patients. Objective or quantifiable items may thus be retained optionally in the CEF (items related to signs and symptoms were already optional in the SRQ [19]).

Considering the reliability of most patient-reported data and the associated limitations, and given that no effect of gender, age, education level, or disease severity was observed, the registry forms have been modified to increase concordance and efficiency. In particular, the formulation of questions has been optimized; patient feedback helped in this task. Since we established that the registry can rely more on patient-reported data, the CEF contents were revised to bring the focus on discordant items.

\section{Patient-related outcome measures are key to further research}

The present study validates and reinforces the French registry philosophy: recording the patient and physician complementary visions is invaluable to warrant the data quality expected to lead relevant statistical analysis, in particular based on ML techniques. The natural course of FSHD is highly variable and predicting disease outcomes is not yet achievable. The so-called ReSolve clinical study (NCT03458832) has been set up as a way to identify novel PROMs and expand knowledge on the natural history of FSHD [31]. Registries are instrumental in collecting PROMs $[6,32,33]$ as they are an increasingly important key feature of clinical trials [34]. The combination of patient- and physician-reported data in the French FSHD registry will be a significant asset in gathering the data necessary to define the objectives and outcome measures, and fine-tune the eligibility criteria, of future clinical trials.

\section{Building predictive models}

Alternatively, and complementarily, applying ML on the registry data could help better characterize the stages of disease progression and make individualized predictions. Furthermore, it is believed that AI will play a fundamental role in finding treatments for rare diseases [35]. ML has recently started to be implemented in the diagnostic process of some neuromuscular diseases, by improving the analysis of electromyograms [36] or MRI patterns [37]. It is also used in the context of autoimmune diseases, including neuromuscular disorders such as myasthenia gravis, to help predict the disease outcomes [38]. By pooling national datasets, thereby vastly increasing the data available, the global FSHD project [39] would greatly potentiate the efficiency of AI analyses. The hindrance towards establishing a predictive FSHD model may be more of an administrative and regulatory nature than of a scientific one.

\section{Conclusions}

This study showed that patient-reported data are as reliable as physician-made assessments on condition that they are objective or quantifiable, which includes, surprisingly, the Brooke and Vignos scores. This finding helped optimize the forms used in the French FSHD registry, which will be a key resource for designing future therapeutic trials and improving standards of care, in part through the development of PROMs. As the outcome of telemedicine consultations in France, and other parts of the world, in the wake of the Covid-19 pandemic showed, the identification of reliable PROMs is at the heart of the future of medical practice. Achieving high-quality FSHD data is thus all the more important. However, extracting relevant new information, through e.g. ML techniques, may require pooling out resources from several registries. In this view, performing the same comparative analysis on data from patient- and clinician-based registries would be an interesting follow-up study and could benefit the 


\section{harmonization registries require to bring out relevant and usable PROMs.}

\begin{abstract}
Abbreviations
AFM-Téléthon: French Muscular Dystrophy Association; Al: Artificial intelligence; CCTIRS: French Advisory Committee on data processing in health research; CEF: Clinical evaluation form; CNIL: French Data Protection Authority; FSHD: Facioscapulohumeral muscular dystrophy; FSHD1: FSHD type 1; FSHD2: FSHD type 2; ICC: Intra-class coefficient; ML: Machine learning; PROM: Patientreported outcome measure; SMCHD1: Structural maintenance of chromosomes flexible hinge domain containing 1; SRQ: Self-report questionnaire; VAPS: Visual Analog Pain Scale.
\end{abstract}

\section{Supplementary Information}

The online version contains supplementary material available at https://doi. org/10.1186/s13023-021-01793-6.

Additional file 1. Figure S1: Agreement (in Kappa or ICC values) between item answers in the SRQ and the CEF in the younger subgroup of the cohort ( $<65$ years; $N=209$ ).

Additional file 2. Figure S2: Agreement (in Kappa or ICC values) between item answers in the SRQ and the CEF in the older subgroup of the cohort ( $\geq 65$ years, $N=209$ ).

Additional file 3. Figure S3: Agreement (in Kappa or ICC values) between item answers in the SRQ and the CEF in the subgroup associated with less severe forms of the disease (CSS $<6, N=72$ ).

Additional file 4. Figure S4: Agreement (in Kappa or ICC values) between item answers in the SRQ and the CEF in the subgroup associated with more severe forms of the disease ( $C S S \geq 6 ; N=165$ ).

Additional file 5. Figure S5: Agreement (in Kappa or ICC values) between item answers in the SRQ and the CEF in the subgroup associated with men.

Additional file 6. Figure S6: Agreement (in Kappa or ICC values) between item answers in the SRQ and the CEF in the subgroup associated with women.

Additional file 7. Figure S7: Agreement (in Kappa or ICC values) between item answers in the SRQ and the CEF in the subgroup associated with preFrench secondary education degree.

Additional file 8. Figure S8: Agreement (in Kappa or ICC values) between item answers in the SRQ and the CEF in the subgroup associated with post- French secondary education degree.

\section{Acknowledgements}

The authors want to thank the patients for their strong involvement in this project. We are grateful to Isabelle Pélisson and Jonathan Pini for their critical reading of the manuscript and their insightful suggestions.

The French FSHD registry collaboration group: Blandine Acket (CHU Toulouse, Toulouse, France); Jean-Christophe Antoine (CHU, Saint-Étienne, Saint-Étienne, France); Shahram Attarian (AP-HM, Marseille, France); Guillaume Bassez (Institut de Myologie, Paris, France); Anne-Laure Bédat-Millet (CHU Rouen, Rouen, France); Anthony Béhin (Institut de Myologie, Paris, France); Rémi Bellance (CHU Martinique, Fort-de-France, France); Michela Bisciglia (Institut de Myologie, Paris, France); Véronique Bombart (CHU Reims, Reims, France); Rosalie Boitet (CHU Montpellier, Montpellier, France); Pascale Bonnet (CHU Grenoble, Grenoble, France); Françoise Bouhour (Hospices Civils de Lyon, Lyon, France); Célia Boutte (CHU Grenoble); Brigitte Chabrol (AP-HM, Marseille, France); JeanBaptiste Chanson (CHRU Strasbourg, Strasbourg, France); Françoise Chapon (CHU Caen, Caen, France); Ariane Choumert (CHU La Réunion, Saint-Pierre, France); Pauline Coignard (Centre mutualiste de Kerpape, Ploemeur, France); Jean-Yves Cornu (CHU Besançon, Besançon, France); Benoît Daubail (CHU Dijon); Elisa De La Cruz (CHU Montpellier, Montpellier, France); Léa Declerck (CHU La Réunion, Saint-Pierre, France); Capucine Delattre (Hospices Civils de Lyon, Lyon, France); Florence Demurger (CH Bretagne-Atlantique, Vannes,
France); Véronique Dulieu (CHU Dijon, Dijon, France); Aurélie Duruflé (Pôle Saint-Hélier, Rennes, France); Fanny Duval (CHU Bordeaux, Bordeaux, France); Florence Esselin (CHU Montpellier, Montpellier, France); Teresinha Evangelista (Institut de Myologie, Paris, France); Bruno Eymard (Institut de Myologie, Paris, France); Anthony Faivre (HIA Sainte-Anne, Toulon, France); Léonard Féasson (CHU, Saint-Étienne, Saint-Étienne, France); Xavier Ferrer (CHU Bordeaux, Bordeaux, France); François Feuvrier (CHU Montpellier, Montpellier, France); Olivier Flabeau (CH Côte Basque, Bayonne, France); Mélanie Fradin (CHU Rennes, Rennes, France); Alain Furby (CHU Saint-Étienne, Saint-Étienne, France); Jérémy Garcia (CHU Nice, Nice, France); Hélène Gervais-Bernard (Hospices Civils de Lyon, Lyon, France); Teresa Gidaro (Institut de Myologie, Paris, France); Karima Ghorab (CHU Limoges, Limoges, France); Marc Jeanpierre (Institut Cochin, Paris, France); Hubert Journel (CH Bretagne-Atlantique, Vannes, France); Arnaud Lacour (CHU Lille, Lille, France); Pascal Laforêt (Hôpital Raymond-Poincaré, Garches, France); Emmeline Lagrange (CHU Grenoble, Grenoble, France); Valérie Layet (GH du Havre, Le Havre, France); Gérard Leclaire (Centre mutualiste de Kerpape, Ploemeur, France); Jean-Luc Le Guiet (Centre mutualiste de Kerpape, Ploemeur, France); Gwenaël Le Guyader (CHU Poitiers, Poitiers, France); François Leroy (CHU Caen, Caen, France); France Leturca (Institut Cochin, Paris, France); Nicolas Lévy (AP-HM, Marseille, France); Sarah Léonard-Louis (Institut de Myologie, Paris, France); Laurent Magy (CHU Limoges, Limoges, France); Edoardo Malfatti (Hôpital Henri-Mondor, Créteil, France); Marion Masingue (Institut de Myologie, Paris, France); Gilles Mazaltarine (Hôpital Henri-Mondor, Créteil, France); Dominique Ménard (CHU Rennes, Rennes, France); Maud Michaud (CHU Nancy, Nancy, France); Marie-Christine Minot-Myhié (CHU Rennes, Rennes, France); Marie-Doriane Morard (Hospices Civils de Lyon, Lyon, France); Juliette Nectoux (Institut Cochin, Paris, France); Karine Nguyen (AP-HM, Marseille, France); Julie Nicomette (CHU Reims, Reims, France); Jean-Baptiste Noury (CHRU Brest, Brest, France); Sybille Pellieux (CHRU Tours, Tours, France); Laetitia Percebois-Macadré (CHU Reims, Reims, France); Yann Péréon (CHU Nantes, Nantes, France); Solange Perrin-Callot (CH Émile Muller, Mulhouse, France); Philippe Petiot (Hospices Civils de Lyon, Lyon, France); Sylviane Peudenier (CHRU Brest, Brest, France); Bénédicte Pontier (CHU Clermont-Ferrand, Clermont-Ferrand, France); Florence Portet (CHU Montpellier, Montpellier, France); Jean Pouget (AP-HM, Marseille, France); Marguerite Preudhomme (CHU Lille, Lille, France); Hélène Rauscent (CHU Rennes, Rennes, France); Dimitri Renard (CHU Nîmes, Nîmes, France); Audrey Riou (CHU Rennes, Rennes, France); François Rivier (CHU Montpellier, Montpellier, France); Emmanuelle Salort-Campana (AP-HM, Marseille, France); Stéphane Schaeffer (CHU Caen, Caen, France); Jean-Philippe Simon (CHU Caen, Caen, France); Aurélie Siri (CHU Nancy, Nancy, France); Marco Spinazzi (CHU Angers, Angers, France); Tanya Stokovic (Institut de Myologie, Paris, France); Juliette Svahn (Hospices Civils de Lyon, Lyon, France); François Tabaraud (CHU Limoges, Limoges, France); Frédéric Taithe (CHU Clermont-Ferrand, Clermont-Ferrand, France); Céline Tard (CHU Lille, Lille, France); Christel Thauvin (CHU Dijon, Dijon, France); Philippe Thoumie (Hôpital Rotschild, Paris, France); Claire-Lise Tournier-Gervason (Chambéry, France); Christine Tranchant (CHRU Strasbourg, Strasbourg, France); Jon Andoni Urtizberea (Institut de Myologie, Paris, France); Christophe Vial (Hospices Civils de Lyon, Lyon, France); Michel Vidaud (Institut Cochin, Paris, France); Fabien Zagnoli (CHRU Brest, Brest, France).

\section{Authors' contributions}

BS helped design the study and collect data, contributed to data interpretation, drafted and revised the manuscript. CS analyzed and interpreted the data and contributed to draft and revise the manuscript. CG contributed to design the study, prepared the data, and revised the manuscript. SR and RB helped design the study and collect data. CE helped design the study. LV, PC GSVT, AEL, AM, RJM, FB, ANP, AJP, SS, and the French FSHD registry collaboration group collected the data. CB and SS conceived and designed the study, contributed to draft and critically reviewed the manuscript. All authors read and approved the final manuscript.

\section{Funding}

This work was supported by the AFM-Téléthon (Grant Number DMFSH802).

\section{Availability of data and materials}

Data are accessible on the registry website, http://www.fshd.fr, through log-in credentials for protecting personal privacy and proprietary information. However, the aggregated data supporting the findings of this study can be obtained upon reasonable request to the French FSHD registry steering committee. 


\section{Declarations}

\section{Ethics approval and consent to participate}

The registry was approved by the French data protection authority $(C N I L$; Authorization Number 912291) and the French advisory committee on data processing in health research (CCTIRS; Favorable Opinion Number 12.004bis).

\section{Consent for publication}

Written consents have been obtained from all patients to use and store their data in the French FSHD registry.

\section{Competing interests}

The authors declare that they have no competing interests.

\section{Author details}

${ }^{1}$ Université Côte d'Azur, Service Système Nerveux Périphérique \& Muscle, Centre Hospitalier Universitaire de Nice, Nice, France. ${ }^{2}$ Medical Affairs Department, AFM-Telethon, Evry, France. ${ }^{3}$ Aix Marseille Univ, INSERM, MMG, Bioinformatics and Genetics, Marseille, France. ${ }^{4}$ APHM, Hôpital Timone Enfants, Laboratoire de Génétique Moléculaire, Marseille, France. ${ }^{5}$ Department of Neurology, Toulouse University Hospital, Toulouse, France. ${ }^{6}$ Centre de Référence des Maladies Neuromusculaires AOC, Hôpital Pellegrin, CHU de Bordeaux, Bordeaux, France. ${ }^{7}$ Centre de Référence des Maladies Neuromusculaires, Service de Médecine Physique et de Réadaptation, CHU de Lille, Lille, France. ${ }^{8}$ Department of Neurology, APHP, Bicêtre University Hospital, Le Kremlin-Bicêtre, France. ${ }^{9}$ French National Reference Center for Rare Neuropathies (NNERF), LeKremlin-Bicêtre, France. ${ }^{10}$ INSERM U1195 and Paris-Saclay University, Le Kremlin-Bicêtre, France. ${ }^{11}$ Referral Center for Neuromuscular Diseases Atlantique-Occitanie-Caraïbes, CHU Nantes, Nantes, France. ${ }^{12}$ Département de Neurologie, CHU de Montpellier, Montpellier, France. ${ }^{13}$ NMD Reference Center, Reims Champagne-Ardenne University Hospital, EA3797, Reims, France. ${ }^{14}$ Centre de Référence des Maladies Neuromusculaires Atlantique-Occitanie-Caraïbes, FILNEMUS, Service de Neurologie, CHU d'Angers, Angers, France. ${ }^{15}$ Centre de Référence des Maladies Neuromusculaires Nord/Est/Ile-de-France, Service de Neurologie, Hôpitaux Universitaires de Strasbourg, Strasbourg, France. ${ }^{16}$ Department of Clinical Neurophysiology, CHU Dijon Bourgogne, Dijon, France. ${ }^{17}$ Institute for Research on Cancer and Aging of Nice (IRCAN), INSERM U1081, CNRS UMR 7284, Faculté de Médecine, Université Côte d'Azur (UCA), Nice, France.

Received: 24 December 2020 Accepted: 30 March 2021

Published online: 02 March 2022

\section{References}

1. Kinoshita J. Dogs destined to develop muscular dystrophy evade their genetic fate. FSH Society website. Published November 17, 2015. https:// www.fshsociety.org/2015/11/dogs-destined-to-develop-muscular-dystr ophy-evade-their-genetic-fate/. Accessed 15 Jan 2019.

2. Flanigan KM et al. Genetic characterization of a large, historically significant Utah kindred with facioscapulohumeral dystrophy. Neuromuscul Disord. 2001:11(6-7):525-9.

3. Deenen JC, Arnts H, van der Maarel SM, Padberg GW, Verschuuren JJ, Bakker E et al. Population-based incidence and prevalence of facioscapulohumeral dystrophy. Neurology. 2014;83(12):1056-9.

4. Mostacciuolo ML, Pastorello E, Vazza G, Miorin M, Angelini C, Tomelleri $G$ et al. Facioscapulohumeral muscular dystrophy: epidemiological and molecular study in a north-east Italian population sample. Clin Genet. 2009;75(6):550-5

5. Lunt PW, Harper PS. Genetic counselling in facioscapulohumeral muscu lar dystrophy. J Med Genet. 1991;28(10):655-64.

6. Evangelista T, Wood L, Fernandez-Torron R, Williams M, Smith D, Lunt P, et al. Design, set-up and utility of the UK facioscapulohumeral muscular dystrophy patient registry. J Neurol. 2016;263:1401-8.

7. Padberg GW. Facioscapulohumeral disease. Leiden: Faculty of Medicine Leiden University Medical Center (LUMC), Leiden University; 1982.

8. Statland JM, Tawil R. Risk of functional impairment in facioscapulohumeral muscular dystrophy. Muscle Nerve. 2014:49(4):520-7.
9. Funakoshi M, Goto K, Arahata K. Epilepsy and mental retardation in a subset of early onset 4q35-facioscapulohumeral muscular dystrophy. Neurology. 1998;50(6):1791-4

10. Chen TH, Lai YH, Lee PL, Hsu JH, Goto K, Hayashi YK et al. Infantile facioscapulohumeral muscular dystrophy revisited: expansion of clinical phenotypes in patients with a very short EcoRI fragment. Neuromuscul Disord. 2013:23(4):298-305.

11. Statland JM, Sacconi S, Farmakidis C, Donlin-Smith CM, Chung M, Tawil R. Coats syndrome in facioscapulohumeral dystrophy type 1: frequency and D4Z4 contraction size. Neurology. 2013;80(13):1247-50.

12. Brouwer OF, Padberg GW, Ruys CJ, Brand R, Grote JJ. Hearing loss in facioscapulohumeral muscular dystrophy. Neurology. 1991;41(12):1878.

13. Lemmers RJ, Tawil R, Petek LM, Balog J, Block GJ, Santen GW et al. Digenic inheritance of an SMCHD1 mutation and an FSHD-permissive D4Z4 allele causes facioscapulohumeral muscular dystrophy type 2. Nat Genet. 2012:44(12):1370

14. Sacconi S, Briand-Suleau A, Gros M, Baudoin C, Lemmers RJ, Rondeau S et al. FSHD1 and FSHD2 form a disease continuum. Neurology. 2019;92(19):e2273-85.

15. Statland JM, Tawil R. Facioscapulohumeral muscular dystrophy: molecular pathological advances and future directions. Curr Opin Neurol. 2011;24(5):423-8.

16. Wang LH, Tawil R. Facioscapulohumeral dystrophy. Curr Neurol Neurosci Rep. 2016;16(7):66.

17. Rodrigues M, Hammond-Tooke G, Kidd A, Love D, Patel R, Dawkins H, et al. The New Zealand neuromuscular disease registry. J Clin Neurosci. 2012:19(12):1749-50

18. Vercelli L, Mele F, Ruggiero L, Sera F, Tripodi S, Ricci G, et al. A 5-year clinical follow-up study from the Italian National Registry for FSHD. J Neurol. 2021;268:356-66.

19. Guien C, Blandin G, Lahaut P, Sanson B, Nehal K, Rabarimeriarijaona S, et al. The French National Registry of patients with Facioscapulohumeral muscular dystrophy. Orphanet J Rare Dis. 2018;13(1):218.

20. Tawil R, van der Maarel S, Padberg GW, van Engelen BGM. 171st ENMC international workshop: standards of care and management of facioscapulohumeral muscular dystrophy. Neuromuscul Disord. 2010;20:471-5.

21. Brooke MH, Griggs RC, Mendell JR, Fenichel GM, Shumate JB, Pellegrino RJ. Clinical trial in Duchenne dystrophy. I. The design of the protocol. Muscle Nerve. 1981;4(3):186-97

22. Vignos PJ, Spencer GE, Archibald KC. Management of progressive muscular dystrophy of childhood. JAMA. 1963;184(2):89-96.

23. Landis JR, Koch GG. The measurement of observer agreement for categorical data. Biometrics. 1977;33:159-74.

24. Feinstein AR, Cicchetti DV. High agreement but low kappa: I. The problems of two paradoxes. J Clin Epidemiol. 1990;43:543-8.

25. Cicchetti DV, Feinstein AR. High agreement but low kappa: II. Resolving the paradoxes. J Clin Epidemiol. 1990;43:551-8.

26. Koo TK, Li MY. A guideline of selecting and reporting intraclass correlation coefficients for reliability research. J Chiropr Med. 2016;15:155-63.

27. Lunt PW, Compston DA, Harper PS. Estimation of age dependent penetrance in facioscapulohumeral muscular dystrophy by minimising ascertainment bias. J Med Genet. 1989;26:755-60.

28. Ricci E Galluzzi G, Deidda G, Cacurri S, Colantoni L, Merico B, et al. Progress in the molecular diagnosis of facioscapulohumeral muscular dystrophy and correlation between the number of Kpnl repeats at the 4q35 locus and clinical phenotype. Ann Neurol. 1999;45(6):751-7.

29. Tawil R. Chapter 35 - Facioscapulohumeral muscular dystrophy. In: Geschwind DH, Paulson HL, Klein C, editors. Handb Clin Neurol. Amsterdam: Elsevier; 2018. p. 541-8.

30. van der Sluijs BM, Knoop H, Bleijenberg G, van Engelen BG, Voermans NC The Dutch patients' perspective on oculopharyngeal muscular dystrophy: a questionnaire study on fatigue, pain and impairments. Neuromuscul Disord. 2016;26(3):221-6.

31. LoRusso S, Johnson NE, McDermott MP, Eichinger K, Butterfield RJ,

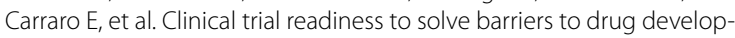
ment in FSHD (ReSolve): protocol of a large, international, multi-center prospective study. BMC Neurol. 2019;19:224. 
32. Sárközy A, Bushby K, Beroud C, Lochmuller H. 157th ENMC international workshop: patient registries for rare, inherited muscular disorders 25-27 January 2008 Naarden, The Netherlands. Neuromuscul Disord NMD. 2008; 18:997-1001.

33. Hamel J, Tawil R. Facioscapulohumeral muscular dystrophy: update on pathogenesis and future treatments. Neurotherapeutics. 2018;15:863-71.

34. Vodicka E, Kim K, Devine EB, Gnanasakthy A, Scoggins JF, Patrick DL. Inclusion of patient-reported outcome measures in registered clinical trials: evidence from ClinicalTrials.gov (2007-2013). Contemp Clin Trials. 2015:43:1-9.

35. Brasil S, Pascoal C, Francisco R, dos Reis Ferreira V, Videira PA, Valadão AG Artificial intelligence (Al) in rare diseases: is the future brighter? Genes. 2019:10:978.

36. Yousefi J, Hamilton-Wright A. Characterizing EMG data using machinelearning tools. Comput Biol Med. 2014;51:1-13.

37. Morrow JM, Sormani MP. Machine learning outperforms human experts in MRI pattern analysis of muscular dystrophies. Neurology. 2020;94:421-2.

38. Stafford IS, Kellermann M, Mossotto E, Beattie RM, MacArthur BD, Ennis S. A systematic review of the applications of artificial intelligence and machine learning in autoimmune diseases. npj Digit Med. 2020;3:1-11.

39. Mul K, Kinoshita J, Dawkins H, van Engelen B, Tupler R, FSHD Consortium. 225th ENMC international workshop: a global FSHD registry framework, 18-20 November 2016, Heemskerk, The Netherlands. Neuromuscul Disord. 2017;27:782-90

\section{Publisher's Note}

Springer Nature remains neutral with regard to jurisdictional claims in published maps and institutional affiliations.

- fast, convenient online submission

- thorough peer review by experienced researchers in your field

- rapid publication on acceptance

- support for research data, including large and complex data types

- gold Open Access which fosters wider collaboration and increased citations

- maximum visibility for your research: over $100 \mathrm{M}$ website views per year

At BMC, research is always in progress.

Learn more biomedcentral.com/submissions 